\title{
Chapter 19. Diabetic patients
}

(C) Japanese Society of Nephrology 2009

- Diabetes is one of the most important target diseases in CKD management.

- Strict glycemic and blood pressure control is essential for suppressing the development and progression of diabetic nephropathy.

- In diabetic nephropathy, strict control of dyslipidemia and other risk factors for CVD is required.

- It has been shown that strict glycemic control can suppress the development of diabetic nephropathy (DCCT, Kumamoto Study).

\section{The target of glycemic control in diabetes}

- Target levels of glycemic control according to the Japan Diabetes Society are shown in Table 19-1.

- The target for HbA1c in diabetic nephropathy is less than $6.5 \%$.

\section{The target of blood pressure control in diabetes}

- Blood pressure control in diabetes is essential similar to glycemic control.

- Target blood pressure is less than $130 / 80 \mathrm{mmHg}$ in diabetes and less than $125 / 75 \mathrm{mmHg}$ in overt diabetic nephropathy.

- Salt intake is restricted to less than $6 \mathrm{~g} /$ day for better blood pressure control.

- ACE inhibitors or ARBs are used as first-line antihypertensive agents, because they are effective in the suppression of new development of diabetes, improvement of proteinuria, and preservation of kidney function.

- If the target blood pressure is not achieved, other antihypertensive agents are concurrently used.

\section{Treatment of diabetes in CKD}

- Diabetes management is principally diet therapy and physical exercise also in CKD. The Guidelines for Education of Daily Life in Diabetic Nephropathy (The Report of the Joint Committee for Diabetic Nephropathy, the Japan Diabetes Society and the Japanese Society of Nephrology, 1999) are shown in Tables 19-2(a, b).

- Due to the high prevalence of diabetic retinopathy, CKD patients with diabetes are consulted by ophthalmologists at the first visit and followed up thereafter.

- Thiazolidine derivatives are not recommended for patients with CKD stage 4-5. Biguanide derivatives are not preferable for CKD stage 3-5 because of possible lactic acidosis.

- If glycemic control is insufficient with oral hypoglycemic agents, insulin therapy is recommended.

- A half-life of insulin is prolonged in CKD with impaired kidney function, which easily causes potential hypoglycemia. Therefore, physicians pay attention to the use of sulfonylurea (SU) derivatives or long-acting insulin.

- Rapid modification of blood glucose may aggravate advanced diabetic retinopathy.

- The serum level of HbA1c or glycoalbumin does not accurately reflect glycemic control status in the 
Table 19-1 Low protein diet for CKD

\begin{tabular}{lllc}
\hline Control & HbA1C $(\%)$ & Fasting blood glucose (mg/dl) & $\begin{array}{c}\text { Blood glucose, 2 h after } \\
\text { meal (mg/dl) }\end{array}$ \\
\hline Excellent & Less than 5.8 & Less than 80-110 & Less than 80-140 \\
Good & Less than 5.8-6.5 & Less than 110-130 & Less than 140-180 \\
Fair & Less than 6.5-7.0 & Less than 130-160 & Less than 180-220 \\
Fair, but not sufficient & Less than 7.0-8.0 & 160 and over & 220 and over \\
Poor & 8.0 and over & & \\
\hline
\end{tabular}

Table 19-2 (a) Lifestyle modification for DM nephropathy (1)

\begin{tabular}{|c|c|c|c|c|c|c|}
\hline Stage & GFR, proteinuria & Daily life & $\begin{array}{l}\text { Total calorie } \\
\text { (kcal/kg/day) }\end{array}$ & $\begin{array}{l}\text { Protein } g / k g \\
\text { weight/day }\end{array}$ & $\begin{array}{l}\text { Salt } \\
\text { g/day }\end{array}$ & $\begin{array}{l}\text { Potassium } \\
\text { (g/day) }\end{array}$ \\
\hline Stage 1 (pre-nephropathy) & $\begin{array}{l}\text { Normal or high, } \\
\text { negative }\end{array}$ & Normal & $25-30$ & - & No restriction & No restriction \\
\hline Stage 2 (early nephropathy) & $\begin{array}{l}\text { Normal or high, } \\
\text { microalbuminuria }\end{array}$ & Normal & $25-30$ & $1.0-1.2$ & No restriction & No restriction \\
\hline $\begin{array}{l}\text { Stage 3A } \\
\text { (overt nephropathy: early) }\end{array}$ & $\begin{array}{l}\geq 60 \mathrm{~mL} / \mathrm{min}, \text { overt } \\
\text { proteinuria }\end{array}$ & Normal & $25-30$ & $0.8-1.0$ & $7-8$ & No restriction \\
\hline $\begin{array}{l}\text { Stage 3B } \\
\quad \text { (overt nephropathy, late) }\end{array}$ & $\begin{array}{l}<60 \mathrm{~mL} / \mathrm{min}, \\
\quad \text { proteinuria } \geq 1 \mathrm{~g} / \text { day }\end{array}$ & $\begin{array}{l}\text { Mild restriction } \\
\text { Avoid overwork }\end{array}$ & $30-35$ & $0.8-1.0$ & $7-8$ & Mild restriction \\
\hline Stage 4 (renal failure) & Azotemia, proteinuria & $\begin{array}{l}\text { Moderate } \\
\text { restriction }\end{array}$ & $30-35$ & $0.6-0.8$ & $5-7$ & 1.5 \\
\hline \multirow[t]{2}{*}{ Stage 5 (dialysis) } & - & Moderate restriction & $\begin{array}{l}\text { Hemodialysis }^{\mathrm{b}} \\
\quad 35-40\end{array}$ & $1.0-1.2$ & $7-8$ & $<1.5$ \\
\hline & & Avoid overwork & CAPD $^{\mathrm{b}} 30-35$ & $1.1-1.3$ & $8-10$ & Mild restriction \\
\hline
\end{tabular}

${ }^{a}$ For hypertension: less than 6 g/day

${ }^{\mathrm{b}}$ Hemodialysis (HD) and continuous ambulatory peritoneal dialysis (CAPD) patients are catabolic. Total calorie intake should be slightly increased compared to DM patients. In CAPD patients, glucose is absorbed from PD fluid. References are the reports to MWL 1992, 1993 and Japan DM Association, 1999 
Table 19-2 (b) Lifestyle modification for DM nephropathy (2)

\begin{tabular}{|c|c|c|c|c|c|}
\hline Stage & Exercise $^{a}$ & Work & House work & $\begin{array}{l}\text { Pregnancy } \\
\text { Delivery }\end{array}$ & Treatment, Diet, Daily life \\
\hline $\begin{array}{l}\text { Stage } 1 \text { (pre- } \\
\text { nephropathy) }\end{array}$ & $\begin{array}{l}\text { - Basically do } \\
\text { exercise for DM }\end{array}$ & - Normal & - Normal & OK & $\begin{array}{l}\text { - Control blood glucose, } \\
\text { Avoid excessive protein intake }\end{array}$ \\
\hline $\begin{array}{l}\text { Stage } 2 \text { (early } \\
\text { nephropathy) }\end{array}$ & $\begin{array}{l}\text { - Basically do } \\
\text { exercise for DM }\end{array}$ & - Normal & - Normal & OK & $\begin{array}{l}\text { - Strict control of blood glucose } \\
\text { - Anti-hypertensive treatment } \\
\text { - Avoid excessive protein intake }\end{array}$ \\
\hline $\begin{array}{l}\text { Stage 3A (overt } \\
\text { nephropathy: early) }\end{array}$ & $\begin{array}{l}\text { - Basically exercise } \\
\text { is OK } \\
\text { - Amount of exercise } \\
\text { is dependent of the } \\
\text { condition } \\
\text { - Stop excess exercise }\end{array}$ & • Normal & - Normal & Not allowed & $\begin{array}{l}\text { - Strict control of blood glucose } \\
\text { - Anti-hypertensive treatment } \\
\text { - Protein restriction }\end{array}$ \\
\hline $\begin{array}{l}\text { Stage 3B (overt } \\
\text { nephropathy: late) }\end{array}$ & $\begin{array}{l}\text { - Restrict exercise } \\
\text { - Slight exercise to } \\
\text { maintain physical } \\
\text { strength }\end{array}$ & $\begin{array}{l}\text { - Restrict exercise } \\
\text { - Normal } \sim \text { slight } \\
\text { restriction, depend } \\
\text { on the job }\end{array}$ & $\begin{array}{l}\text { - Mild restriction } \\
\text { - Work up to feel } \\
\text { fatigue }\end{array}$ & Not allowed & $\begin{array}{l}\text { - Control of blood glucose } \\
\text { - Anti-hypertensive treatment, } \\
\text { protein restriction }{ }^{\mathrm{b}} \\
\text { - Water intake should be } \\
\text { determined with the degree } \\
\text { of edema and congestive } \\
\text { heart failure }\end{array}$ \\
\hline Stage 4 (renal failure) & $\begin{array}{l}\text { - Restrict exercise } \\
\text { - Walking or warm-up } \\
\text { exercise is OK }\end{array}$ & $\begin{array}{l}\text { - Slight restriction } \\
\sim \text { restrict job } \\
\text { - Avoid fatigue } \\
\text { - Stop over-work, } \\
\text { No night shift }\end{array}$ & $\begin{array}{l}\text { - Restricted } \\
\text { - Not overwork: } \\
\text { feel no fatigue }\end{array}$ & Not allowed & $\begin{array}{l}\text { - Control of blood glucose and } \\
\text { hypertension } \\
\text { - Low protein } \text { diet }^{\mathrm{b}} \text { (until } \\
\text { dialysis) } \\
\text { - Water intake should be } \\
\text { determined with the degree } \\
\text { of edema and congestive } \\
\text { heart failure }\end{array}$ \\
\hline Stage 5 (Dialysis) & $\begin{array}{l}\text { - Basically slight } \\
\text { exercise only } \\
\text { - Stop excess exercise }\end{array}$ & $\begin{array}{l}\text { - Basically, mile } \\
\text { restricted work } \\
\text { - Avoid overwork, } \\
\text { Restrict extra-work }\end{array}$ & $\begin{array}{l}\text { - Normal } \\
\text { - Not overwork: } \\
\text { feel no fatigue }\end{array}$ & Not allowed & $\begin{array}{l}\text { - Control of blood glucose and } \\
\text { hypertension } \\
\text { - Dialysis or renal } \\
\text { transplantation } \\
\text { - Restrict water intake } \\
\text { (inter-dialytic weight gain: } \\
\text { less than 5\% of ideal weight) }\end{array}$ \\
\hline
\end{tabular}

${ }^{a}$ Degree of restriction is dependent on proteinuria or hypertension. Restrict hard exercise if proliferative retinopathy is present regardless of the stage of CKD

b Use "Food exchange table" for diabetes and diabetic nephropathy

presence of anemia or hypoalbuminemia, respectively. The HbA1c level may be underestimated in the shortened lifespan of red blood cells or in the use of erythropoiesis- stimulating agents. Caution is therefore taken in the evaluation of HbAlc or glycoalbumin when CKD is associated with anemia or hypoalbuminemia. 